\title{
Datos morfométricos de Trypanosoma evansi en carpinchos (Hydrochaeris hydrochaeris) de Chaco, Argentina
}

\author{
Santa Cruz, A.; Comolli, J.; Ortiz, J.; González, J.; González, A. \\ Servicio de Producciones No Tradicionales, Facultad de Ciencias Veterinarias, UNNE. Sargento Cabral 2139, \\ Corrientes (3400), Argentina. Tel/Fax: (54) 379-4425753. E-mail: jacomolli@vet.unne.edu.ar.
}

\begin{abstract}
Resumen
Santa Cruz, A.; Comolli, J.; Ortiz, J.; González, J.; González, A.: Datos morfométricos de Trypanosoma evansi en carpinchos (Hydrochaeris hydrochaeris) de Chaco, Argentina. Rev. vet. 24: 1, 60-62, 2013. El objetivo del trabajo fue verificar la existencia de variaciones morfométricas de Trypanosoma evansi en Hydrochaeris hydrochaeris criados en cautiverio en Chaco, Argentina. Para ello se efectuaron mediciones de variables biométricas de 52 ejemplares de $T$. evansi provenientes de carpinchos. Se reconocieron dos variaciones morfométricas del parásito, delgadas y rechonchas. Las dimensiones de ambas formas se compararon con los resultados obtenidos en T. evansi de carpinchos del amazonas peruano. Los resultados revelaron diferencias significativas en todas las variables sometidas a estudio $(\mathrm{p}<0,05)$. Se concluye que al parasitar a H. hydrochaeris, T. evansi presenta dimensiones morfométricas diferentes a las halladas en Perú, variando inclusive en el mismo animal.
\end{abstract}

Palabras clave: Trypanosoma evansi, Hydrochaeris hydrochaeris, dimensiones.

\begin{abstract}
Santa Cruz, A.; Comolli, J.; Ortiz, J.; González, J.; González, A.: Morphometric data of Trypanosoma evansi in capybaras (Hydrochaeris hydrochaeris) from Chaco, Argentina. Rev. vet. 24: 1, 60-62, 2013. The aim of this study was to report morphometric variation of Trypanosoma evansi in Hydrochaeris hydrochaeris bred in captivity in Chaco, Argentina. Biometric measurement was performed on 52 dimensions of $T$. evansi parasitizing capybaras. T. evansi was present in two different forms, slender and stumpy. Dimensions in both forms were compared with those found in T. evansi parasitizing H. hydrochaeris from the Peruvian Amazonas. Results showed significant differences $(\mathrm{p}<0,05)$ in all the variables under study. It is concluded that $T$. evansi parasitizing H. hydrochaeris from Chaco, Argentina, presents different morphometric dimensions when compared to the same parasite in Peru, showing variations even in the same animal.
\end{abstract}

Key words: Trypanosoma evansi, Hydrochaeris hydrochaeris, dimensions.

\section{INTRODUCCIÓN}

Trypanosoma evansi es un protozoario flagelado que afecta a especies domésticas como equinos, bovinos y perros, así como a animales silvestres como el coatí (Nasua nasua), carpincho o capibara (Hydrochaeris hydrochaeris) ${ }^{6,8-10}$ y el vampiro (Desmodus rotundus $)^{4}$, los que cumplen el rol de potenciales hospedadores reservorios. Existen fuertes evidencias que sugieren que el inicio del "mal de caderas" equino, causado por T. evansi se debe a factores como la presencia de animales domésticos y silvestres afectados, además de altas concentraciones de vectores ${ }^{2}$.

Recibido: 16 octubre 2012 / Aceptado: 19 noviembre 2012 Presentado en Reunión SGCYT-UNNE 2003
Los trypanosomas son pleomórficos y poseen formas intermedias delgadas, formas delgadas y formas rechonchas, siendo éstas últimas observadas en menor proporción $^{7}$. El kinetoplasto está ausente en muchos de ellos ${ }^{6}$ y en aislamientos de Pantanal (Brasil) se demostraron variaciones biométricas significativas ${ }^{7}$.

El objetivo de este trabajo fue reportar las variaciones morfométricas halladas en T. evansi parasitando H. hydrochaeris criados en cautiverio en Chaco, Argentina.

\section{MATERIAL Y MÉTODOS}

Se dispuso de 7 hembras adultas procedentes de un criadero de La Eduvigis (Chaco, Argentina), ubicado a $26,7^{\circ}$ Sur y $59,1^{\circ}$ Oeste. Un ejemplar reveló signos clínicos compatibles con los de trypanosomosis, otro 
registraba antecedentes de aborto y los demás no manifestaron ninguna sintomatología.

Se procedió a la extracción de sangre por punción de la vena femoral, con la cual se efectuaron frotis coloreados con Giemsa. Las observaciones se realizaron con microscopio óptico y objetivo de inmersión (100x). Se establecieron las características biométricas de un total de 52 hemoflagelados. Para determinar la especie se tuvieron en cuenta las características morfológicas corrientemente utilizadas (presencia o ausencia de kinetoplasto subterminal, desarrollo de la membrana ondulante y del flagelo libre) $5,7,10,11$.

Se calcularon la media aritmética y el desvío estándar (DE) para las siguientes variables biométricas (Figura 1): PN: longitud desde el extremo posterior a la mitad del núcleo; AN: longitud desde el extremo anterior a la mitad del núcleo; F: longitud del flagelo libre; L: longitud total, NI: índice nuclear (cociente entre PN y AN). Estos datos fueron comparados con las mismas dimensiones obtenidas en sangre de carpinchos en cautiverio parasitados por $T$. evansi de la región amazónica del Perú ${ }^{5}$, para lo cual se realizó test de t $(\alpha=0,05$; $\mathrm{n}=52$ ) para cada variable.

\section{RESULTADOS Y DISCUSIÓN}

Del total de siete animales muestreados, tres resultaron positivos $(42,86 \%)$ a hemoparásitos, una fue la hembra que había abortado, otra aquélla que presentó signos clínicos de trypanosomosis y muerte posterior, estando la tercera clínicamente sana.

En base a las características morfométricas los hemoparásitos fueron identificados como T. evansi y se
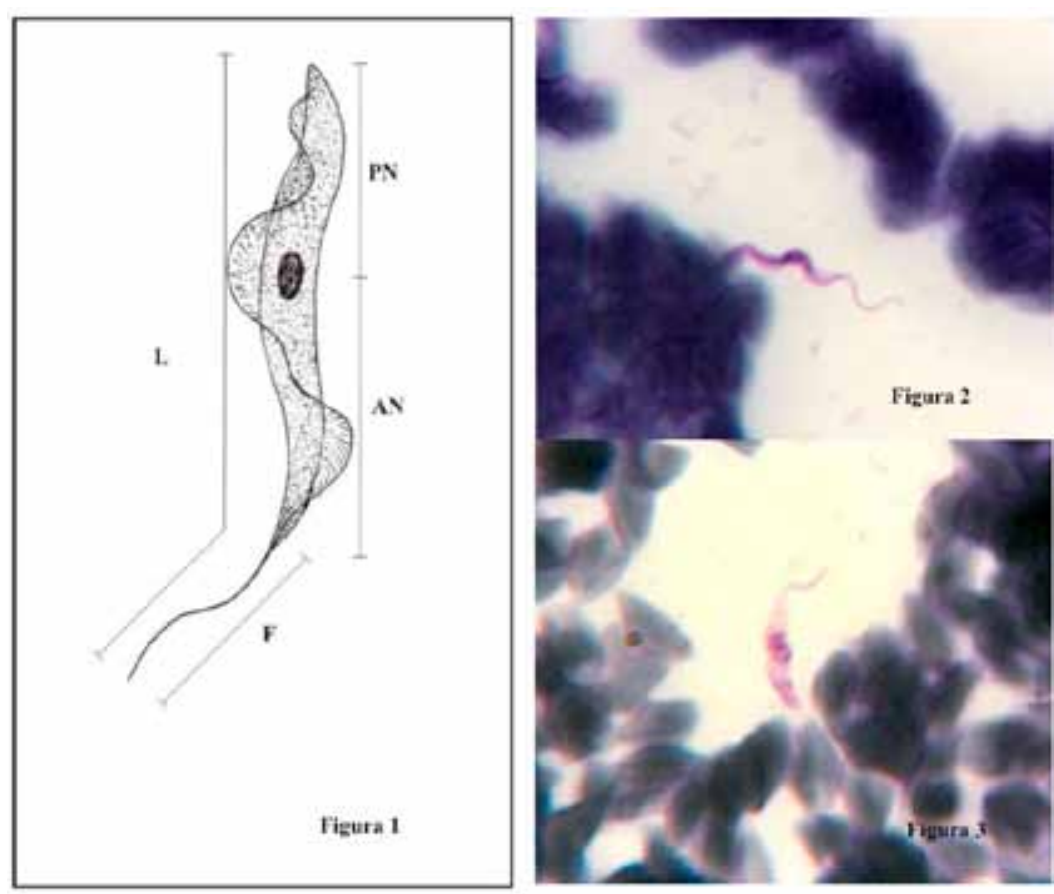

Figura 1. Esquema de T. evansi. PN: longitud del extremo posterior a la mitad del núcleo; AN: longitud del extremo anterior a la mitad del núcleo; F: longitud del flagelo libre; L: longitud total. Figura 2. Forma delgada $T$. evansi, 100x. Figura 3. Forma rechoncha T. evansi, 100x. Chávez ${ }^{5}$.
Tabla 1. Medidas de T. evansi ( $\mu \mathrm{m})$ comparadas con las halladas por Muñoz y Chávez, 2001 (media aritmética $\pm \mathrm{DE})$.

\begin{tabular}{lcc}
\hline variable & Amazonas, Perú & Chaco, Argentina \\
\hline PN & $6,31^{\mathrm{a}} \pm 1,09$ & $8,84^{\mathrm{b}} \pm 1,43$ \\
NA & $4,46^{\mathrm{a}} \pm 1,04$ & $7,11^{\mathrm{b}} \pm 1,71$ \\
F & $6,45^{\mathrm{a}} \pm 1,86$ & $7,25^{\mathrm{b}} \pm 1,66$ \\
L & $17,14^{\mathrm{a}} \pm 2,69$ & $22,24^{\mathrm{b}} \pm 2,39$ \\
NI=PN/NA & $1,46^{\mathrm{a}} \pm 0,29$ & $1,30^{\mathrm{b}} \pm 0,39$ \\
\hline
\end{tabular}

PN: longitud desde el extremo posterior hasta la mitad del núcleo; AN: longitud desde el núcleo hasta el extremo anterior, F: longitud del flagelo libre, L: longitud total, incluyendo el flagelo libre; NI: índice nuclear (cociente entre PN y AN). Letras diferentes para la misma variable implican diferencias significativas $(p<0,05)$.

clasificaron en formas delgadas (Figura 2) y rechonchas (Figura 3). En en ningún caso se observó la presencia de kinetoplasto.

Los resultados de las mediciones se exponen en Tabla 1. De su examen se desprende que los datos obtenidos fueron estadísticamente diferentes $(p<0,05)$ respecto de las observaciones realizadas por Muñoz y

T. evansi ha sido considerado como monomórfico y exclusivamente de forma delgada intermedia ${ }^{1}$, implicando que todas las cepas de parásitos provenientes de diferentes áreas geográficas y distintos hospedadores son morfológicamente indiferenciadas ${ }^{3,4}$. Sin embargo, en un estudio realizado en animales domésticos y silvestres de Pantanal, Brasil, se registraron diferencias morfológicas significativas en algunos ejemplares de $T$. evansi y ausencia de kinetoplasto en la totalidad de los hemoflagelados observados ${ }^{7}$. En equinos y camellos se encontraron formas delgadas y rechonchas con kinetoplasto subterminal ${ }^{11}$. No se reconocieron formas rechonchas en las muestras provenientes de coatí ${ }^{9}$. En gatos se registraron trypomastigotes pleomórficos ${ }^{12}$.

Respecto al polimorfismo y ausencia de kinetoplasto, los resultados obtenidos en esta investigación son coincidentes con los reportados por varios autores $7,11,12$, quienes no solo encontraron diferencias entre distintas especies hospedadoras, sino también entre individuos de la misma especie. Confrontando los resultados del presente estudio con los de la región amazónica de Perú ${ }^{5}$, realizados en carpinchos mantenidos bajo similares condiciones de cautiverio, se hallaron diferencias morfobiométricas evidentes, incluyendo presencia de kinetoplasto $\mathrm{y}$ 
ausencia de formas rechonchas. También debe destacarse que los parásitos del presente trabajo tuvieron un tamaño mayor (Tabla 1).

Este estudio demuestra que los ejemplares de $T$. evansi que parasitan a $H$. hydrochaeris presentan variables morfométricas y biométricas diferentes, inclusive en el mismo animal.

\section{REFERENCIAS}

1. Brun R, Hecker H, Lun Z. 1998. T. evansi and T. equiperdum: distribution, biology, treatment and phylogenetic relationship (a review). Vet Parasitol 79: 95-107.

2. Dávila A, Silva R, Jansen AM. 1996. Dynamics of Trypanosoma evansi outbreaks in the Pantanal, Brazil. FAO Anim Prod \& Health Paper 136: 12-14.

3. Gill BS. 1977. Trypanosomes and trypanosomiasis of indian livestock. Indian Council Agric Res (New Delhi), 4145.

4. Hoare CA. 1972. The trypanosomes of mammals, Blackwell Sci Publ (Zoological Monographs), Oxford (UK), 749 p.

5. Muñoz K, Chávez A. 2001. Trypanosoma evansi isolated from capybara (Hydrochaeris hidrochaeris). Mem Inst Oswaldo Cruz 96: 945-946.

6. Nunes VL, Oshiro ET, Dorval ME, García LA, Da Silva AA, Bogliolo AR. 1993. Investigação epidemiologica sobre Trypanosoma evansi no pantanal sul matogrossense. Estudo de reservatórios. Braz J Vet Parasitol 2: 41-44.
7. Ramírez L, Dávila A, Victoriano A, Silva R, Trajamo V, Jansen A. 1997. Measurement of Trypanosoma evansi isolated from the pantanal. Mem Inst Oswaldo Cruz 92: 483-484.

8. Rodrígues A, Fighera R, Souza T, Schild A, Soares M, Milano J, Barros C. 2005. Surtos de tripanossomíase por T. evansi em eqüinos no Rio Grande do Sul: aspectos epidemiológicos, clínicos, hematológicos e patológicos. Pesq Vet Bras 25: 239-249.

9. Silva R, Arosemena N, Herrera H, Sahib C, Ferreira M. 1995. Outbreak of trypanosomosis due to Trypanosoma evansi in horse of pantanal mato-grossense, Brazil. Vet Parasitol 60: 167-171.

10. Silva RA, Seidl A, Ramirez L, Dávila AM. 2002. Trypanosoma evansi e T. vivax: biologia, diagnóstico e controle, Ed. Embrapa Pantanal, Corumbá (Brasil), 141 p.

11. Tamarit A, Tejedor M, González M, Alberola J, Gutierrez C. 2011. Morphological and biometrical features of Trypanosoma evansi isolates from an outbreak in mainland Spain. Vet Parasitol 177: 152-156.

12. Tarello W. 2005. Trypanosoma evansi infection in three cats. Revue Méd Vét 156: 133-134. 